https://doi.org/10.29013/EJEMS-19-4-80-86

Wang Jasmine,

Westwood High School

E-mail: jasminewang020@gmail.com

\title{
FINANCIAL WORRIES AMONG ADULTS OVER MAINTAINING A STANDARD OF LIVING 2017
}

\begin{abstract}
Aim: This study aims to examine the predictors of adults' financial worries over maintaining a sustainable standard of living in 2017 and to build a predictive model for these financial worries by using an artificial neural network and comparing its performance to a logistic regression model.

Method: Data for the analysis was gathered from the National Health Interview Survey (NHIS) in 2017. All eligible participants from the survey were randomly assigned into 2 groups: the training sample and the testing sample. For both the training sample and testing sample, two models were built - one artificial neural network and one logistic regression for each. Finally, receiver operating characteristics (ROC) were calculated and compared for these two models in order to gauge discrimination capability.

Results: From the data, it can gathered that about $35.8 \%$ of the 26.029 adults experienced financial worries over maintaining standard of living, about $38.2 \%$ among females and $32.9 \%$ among the male.

According to the logistic regression, several variables contributed to likelihood of experiencing financial worries over standard of living. Males were $22.84 \%$ less likely than females to have financial worries over maintaining standard of living. The non-Hispanic adults were $39.51 \%$ less likely to have financial worries over maintaining standard of living than Hispanic adults. The married adults were 14.38\% less likely to have financial worries over maintaining standard of living. The black population was $37.10 \%$ more likely to be worried. Compared to unemployed workers who were still looking for employment, people who were employed or unemployed but not looking were $29.62 \%$ and $48.80 \%$, respectively, less worried. Similarly, according to the neural network, the most important predictors were age, working status, marital status, and race.

For the training sample, the ROC was 0.58 for the logistic regression and 0.66 for the artificial neural network. In the testing sample, the ROC was 0.58 for the logistic regression and 0.65 for the artificial neural network.

Conclusions: In this study, we identified several important predictors for adults' financial worries over maintaining a standard of living in 2017. The most prominent factors were gender, race, marital status and working status. As a culmination of the project, we built a predictive model using both an artificial neural network and a logistic regression to serve as a potential tool for early detection.
\end{abstract}

\section{Keywords:}




\section{Introduction}

Being an essential factor in people's lives, the cost of living represents the amount of money needed to sustain a certain standard of living by affording basic expenses such as housing, food, taxes, and health care $[1]$.

According to a GOBankingRates' analysis of the Bureau of Labor Statistics' Consumer Price Index, the cost of living in America has climbed 14 percent over the past three years. The Consumer Price Index measures the change in prices paid for goods and services including food, shelter, energy, transportation and medical care, all of which are essential for living a sustainable lifestyle [2]. Thus, a rise in the Consumer Price Index could indicate an overall rise in cost of basic living, which could impose an increased burden on significant portions of the population.

With these considerations in mind, this study aims to examine the predictors of adults' financial worries over maintaining standard of living in 2017 and build a predictive model for these financial worries using an artificial neural network and comparing its performance to a logistic regression model.

\section{Data and Methods}

Data

The National Health Interview Survey (NHIS) is the principal source of information on the health of the civilian noninstitutionalized population of the United States. It is one of the major data collection programs of the National Center for Health Statistics (NCHS), which is part of the Centers for Disease Control and Prevention (CDC) [3].

The National Health Interview Survey (NHIS) Data for 2017 was used as the primary source of data in this study.

\section{Models}

We used logistic regression models as one method of calculating the predicted risk. Logistic regression is a part of a category of statistical models called generalized linear models, and it allows one to predict a discrete outcome from a set of variables that may be continuous, discrete, dichotomous, or a com- bination of these. Typically, the dependent variable is dichotomous and the independent variables are either categorical or continuous.

The logistic regression model can be expressed with the formula:

$$
\ln (P / P-1)=\beta_{0}+\beta_{1}^{*} X_{1}+\beta_{2}{ }^{*} X_{2}+\ldots+\beta n^{*} X n
$$

Additionally, a package called "neuralnet" in $R$ was used to conduct neural network analysis. The "neuralnet" package focuses on multi-layer perceptrons which are well applicable when modeling functional relationships.

\section{Variables}

The outcome variable is the percentage of "How worried are you right now about not having enough money for Maintaining Standard of Living?” (ASIRETR)

Table 1.- Variables used in this Study

\begin{tabular}{|l|l|}
\hline \multirow{4}{*}{ SEX } & $1:$ Male \\
ORIGIN_I & $2:$ Female \\
\cline { 2 - 2 } Hispanic Ethnicity & $1:$ Yes \\
& $2:$ No \\
\cline { 2 - 2 } RACRECI3 & $1:$ White \\
& $2:$ Black \\
& $3:$ Asian \\
& $4:$ All other race groups* \\
\cline { 2 - 2 } AGE_P & $1:$ Age $>18$ years old \\
& $2: 0$-17 \\
\cline { 2 - 2 } & $1:$ Northeast \\
& $2:$ Midwest \\
Region & $3:$ South \\
& $4:$ West \\
\hline
\end{tabular}

\section{Results}

According to the data sample, about $35.8 \%$ of the 26.029 adults surveyed had financial worries over maintaining standard of living, about $38.2 \%$ among the female and $32.9 \%$ among the male. Below we have included a thorough description and analysis of the data, providing a more detailed portrayal of the results.

The first model we used to display results in a corrgram. Foundationally, a corrgram is a graphical 
representation of the cells of a matrix of correlations. The graph is intended to display the pattern of correlations in terms of their signs and magnitudes using visual thinning and correlation-based variable ordering. Moreover, the cells of the matrix can be shaded or colored to show the correlation value. In the figure below, the positive correlations are shown in blue, while the negative correlations are shown in red; the darker the hue, the greater the magnitude of the correlation.

Financial Worries Over Maintaining The Current Standard of Living

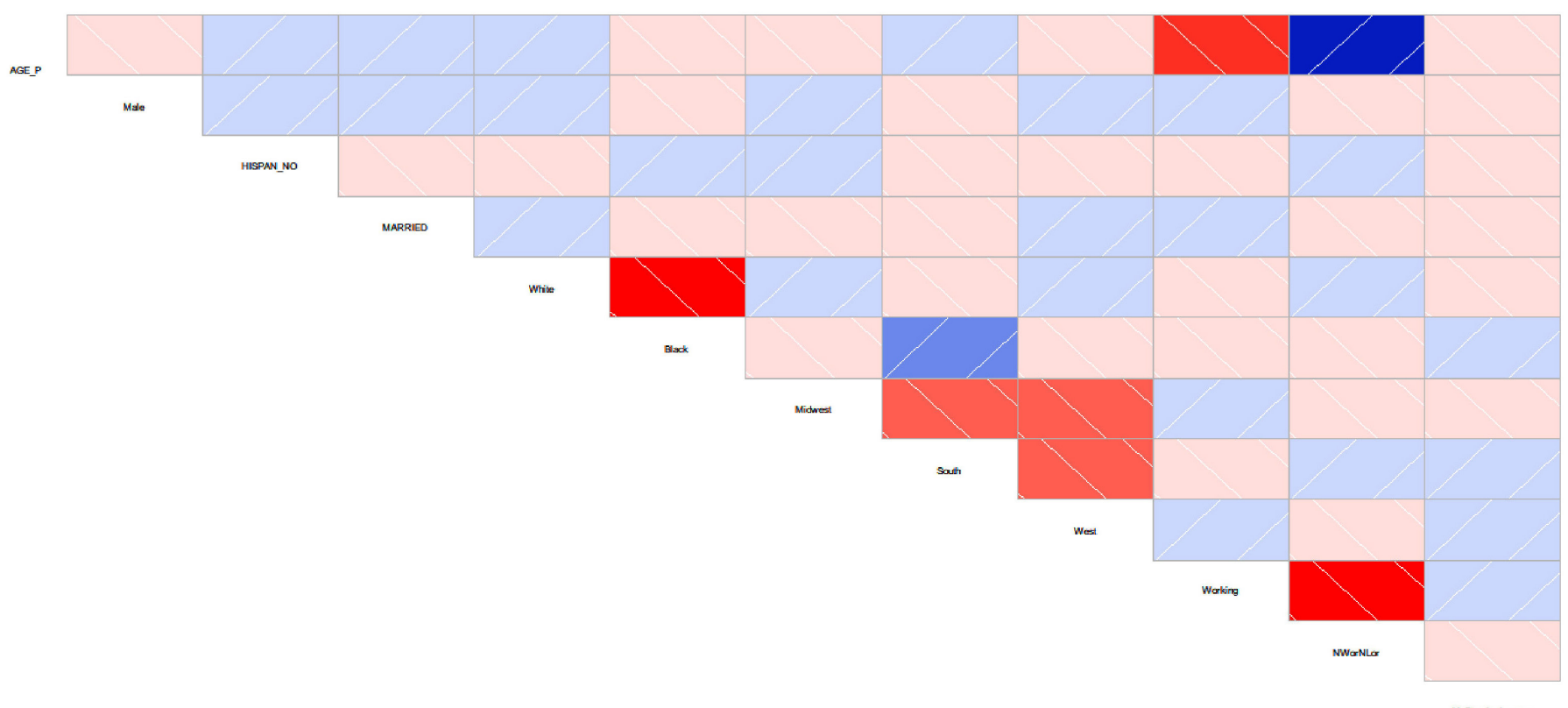

Figure 1. Matrix of Correlations Between Variables

Table 2. - Logistic Regression for Having Financial worries over Maintaining Standard of Living

\begin{tabular}{|c|c|c|c|c|c|}
\hline \multirow[b]{2}{*}{ (Intercept) } & Estimate & Std. Error & z value & $\operatorname{Pr}(>|z|)$ & \\
\hline & 0.361 & 0.091 & 3.946 & 0.000 & *** \\
\hline AGE_P & 0.000 & 0.001 & 0.489 & 0.625 & \\
\hline Male & -0.259 & 0.027 & -9.748 & 0.000 & $* * *$ \\
\hline HISPAN_NO & -0.503 & 0.040 & -12.665 & 0.000 & $* * *$ \\
\hline MARRIED & -0.155 & 0.027 & -5.770 & 0.000 & *** \\
\hline White & 0.074 & 0.052 & 1.419 & 0.156 & \\
\hline Black & 0.316 & 0.064 & 4.934 & 0.000 & *** \\
\hline Midwest & -0.067 & 0.043 & -1.577 & 0.115 & \\
\hline South & 0.015 & 0.039 & 0.391 & 0.696 & \\
\hline West & 0.079 & 0.043 & 1.848 & 0.065 & \\
\hline Working & -0.351 & 0.055 & -6.413 & 0.000 & *** \\
\hline NWorNLor & -0.669 & 0.058 & -11.514 & 0.000 & $* * *$ \\
\hline
\end{tabular}


According to the logistic regression, males were $22.84 \%$ less likely than females to have financial worries over maintaining standard of living. The non-Hispanic adults were $39.51 \%$ less likely to have financial worries over maintaining standard of living than Hispanic adults. The married adults were $14.38 \%$ less likely to have financial worries over maintaining standard of living. The black population was $37.10 \%$ more likely to be worried. Compared to unemployed workers who were still looking for employment, people who were employed or unemployed but not looking were $29.62 \%$ and $48.80 \%$, respectively, less worried. These results can be seen in the data demonstrated by Table 2 and Figure 2.

Table 3.- Odds Ratio and Risk Increase Based on the Logistic Regression

\begin{tabular}{|c|c|c|c|}
\hline & Estimate & Odds Ratio & Risk Increase \\
\hline AGE_P & 0.000 & $100.04 \%$ & $0.04 \%$ \\
\hline Male & -0.259 & $77.16 \%$ & $-22.84 \%$ \\
\hline HISPAN_NO & -0.503 & $60.49 \%$ & $-39.51 \%$ \\
\hline MARRIED & -0.155 & $85.62 \%$ & $-14.38 \%$ \\
\hline White & 0.074 & $107.69 \%$ & $7.69 \%$ \\
\hline Black & 0.316 & $137.10 \%$ & $37.10 \%$ \\
\hline Midwest & -0.067 & $93.51 \%$ & $-6.49 \%$ \\
\hline South & 0.015 & $101.55 \%$ & $1.55 \%$ \\
\hline West & 0.079 & $108.21 \%$ & $8.21 \%$ \\
\hline Working & -0.351 & $70.38 \%$ & $-29.62 \%$ \\
\hline NWorNLor & -0.669 & $51.20 \%$ & $-48.80 \%$ \\
\hline
\end{tabular}

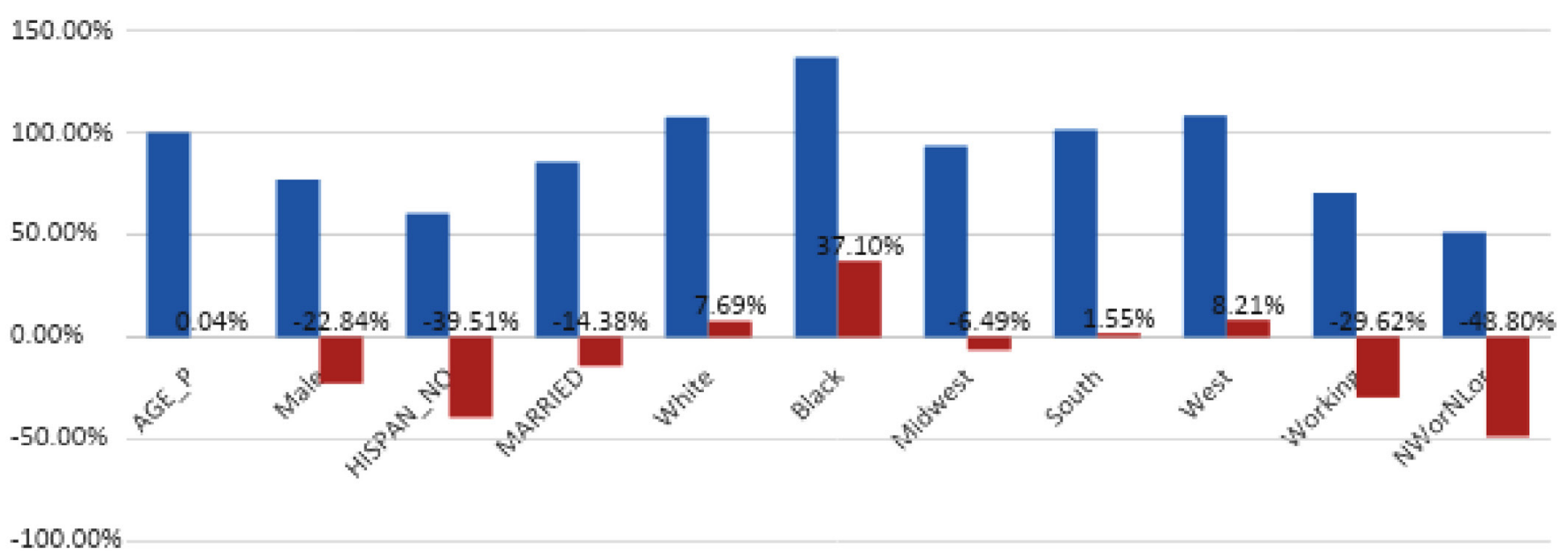

Odds Ratio $\square$ Risk Increase

Figure 2. Odds Ratio and Risk Increase Based on the Logistic Regression 


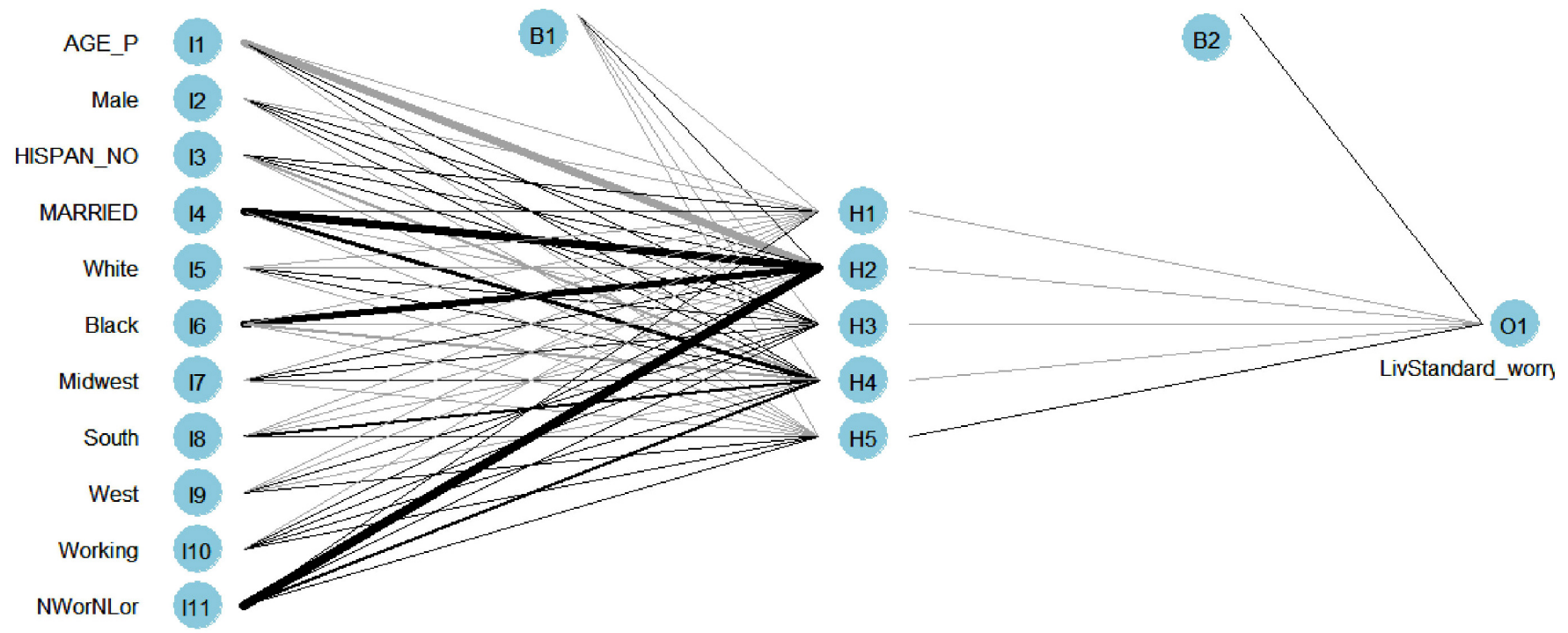

Figure 3. Artificial Neural Network in the training sample

In the above plot, line thickness represents weight magnitude, and line color represents weight sign (black $=$ positive, grey $=$ negative). The net is essentially a black box, so it is difficult to make claims regarding the fitting, the weights and the model. However, it is suffice to say that the training algorithm has converged, and therefore the model is sufficiently ready for use.

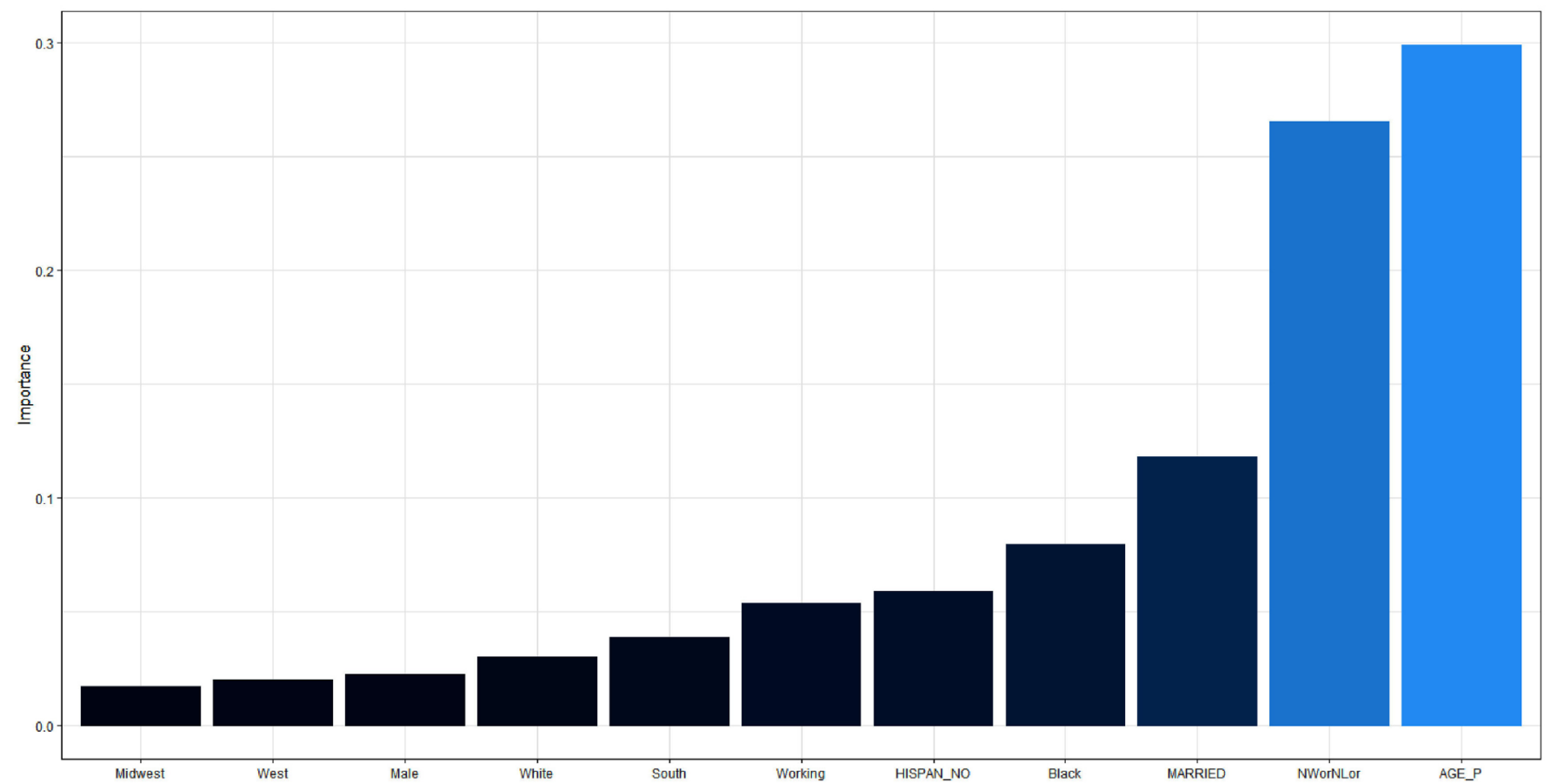

Figure 4. Variable Importance in Artificial Neural Network

As demonstrated by the distributions in most important predictors was age, working status, (Figure 3), according to this neural network, the marital status, and race. 


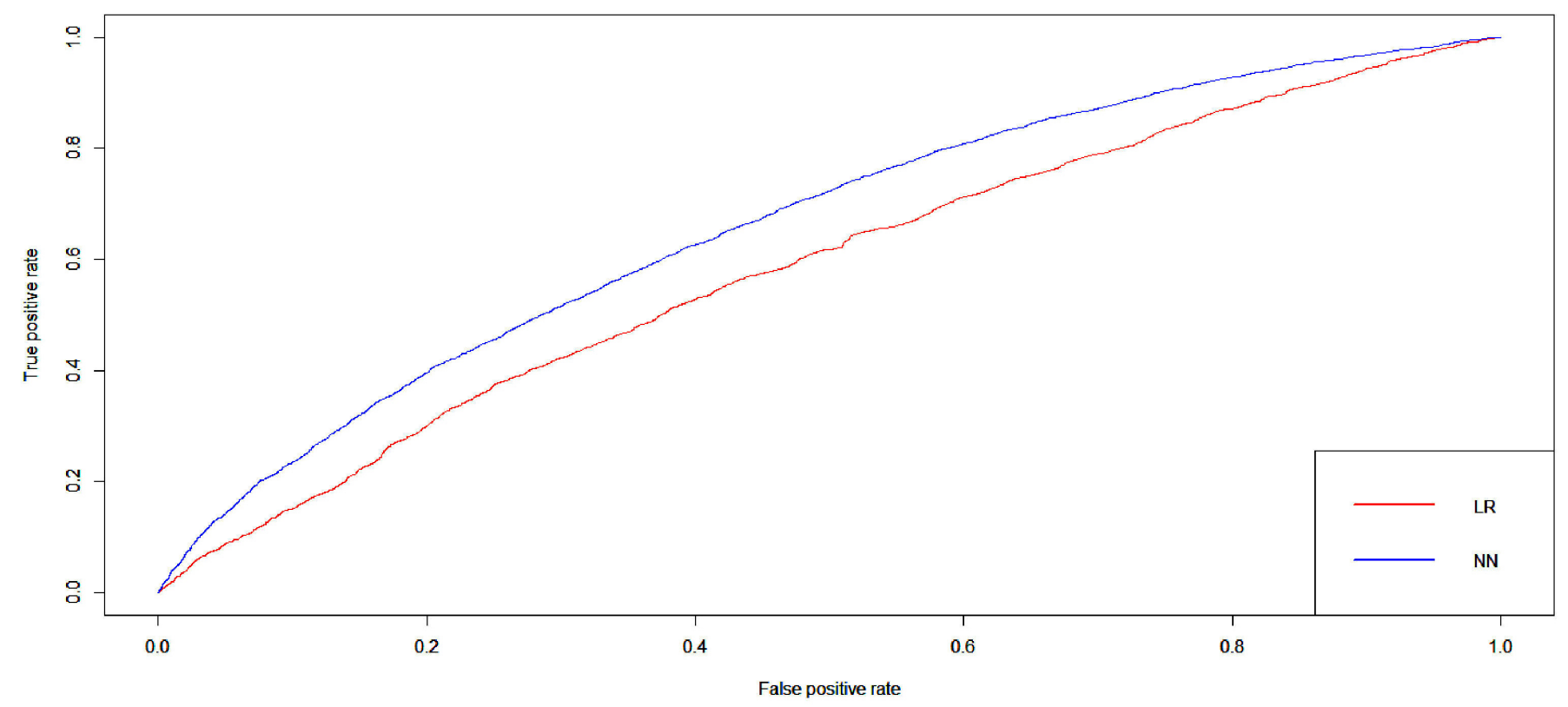

Figure 5. ROC in training sample for Logistic Regression (Red) vs Neural Network (Blue)

Figure 5 reveals the ROC in the training sample testing sample, as demonstrated by (Figure 6) befor both the logistic regression and the neural net- low, the ROC was 0.58 for the Logistic regression work. The ROC was 0.58 for the logistic regression and 0.65 for the artificial neural network. and 0.66 for the artificial neural network. In the

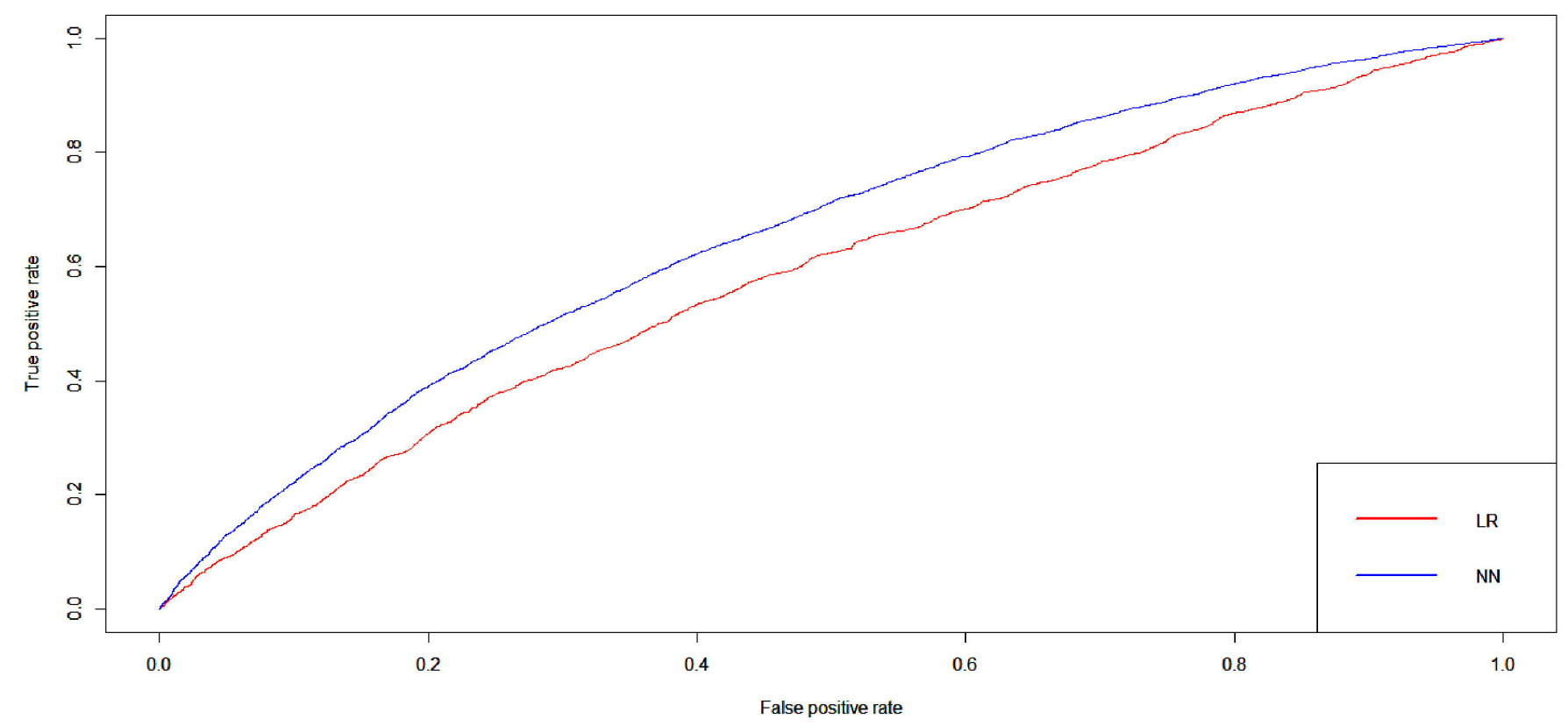

Figure 6. ROC in testing sample for Logistic Regression (Red) vs Neural Network (Blue)

\section{Discussion}

This study aimed to first examine the predictors of adults' financial worries over Maintaining Stan- dard of Living in 2017 and then build a predictive model for adults' financial worries over Maintaining Standard of Living. This involved using an artificial 
neural network and comparing its performance to a logistic regression model.

Upon processing the data, it was revealed that about $35.8 \%$ of the 26.029 adults involved in the data collection had financial worries over maintaining standard of living, about $38.2 \%$ among the female and $32.9 \%$ among the male.

Next, through data analysis, we discovered a range of associations between different factors and likelihood of having worries over maintaining a standard of living. As detailed in the results of this study, according to the logistic regression, factors such as gender, hispanic origins, marital status, and state of employment were key factors in likelihood of having those financial worries. The neural network suggested the involvement of similar factors. According to the network, the most important predictors were age, working status, marital status, and race.

In both the training sample and testing sample, the ROCs were similar for the logistic regression and neural network, suggesting that they both had similar functionality in regards to prediction accuracy. The neural network in both cases had a slightly higher ROC, however, meaning that the network may in fact be slightly more accurate than the regression.

In this study, we identified several important predictors for adults' financial worries over maintaining standard of living in 2017. Some of the most important factors included gender, race, marital status and working status. We built a predictive model using artificial neural network as well as logistic regression to provide a tool for early detection, and discovered that while both have similar degrees of accuracy, the neural network may give a slight advantage.

Using these models, and keeping the relevant factors in mind, perhaps early detection could help identify more potentially prominent areas of need at a faster rate. This way, federal or private organizations seeking to provide assistance to those in financial need could allocate resources more effectively.

\section{References:}

1. Banton, Caroline. "Why the Cost of Living Is Different for Various Cities and Regions." Investopedia, Investopedia, 27 Aug. 2019. URL:http://www.investopedia.com/terms/c/cost-of-living.asp

2. Huddleston, Cameron. "Unemployment Is Low, Wages Are Up - But Cost of Living in America Keeps Rising." GO Banking Rates, Toggle Navigation Back, 18 July 2019. URL:http://www.gobankingrates. com/making-money/economy/rising-cost-of-living-in-america

3. "NHIS - National Health Interview Survey Homepage." Centers for Disease Control and Prevention, Centers for Disease Control and Prevention. URL:http://www.cdc.gov/nchs/nhis/index.htm 